\title{
Complete Response of Locally Advanced Gastric Cancer with Pancreatic Invasion and Gastric Outlet Obstruction after Neoadjuvant Chemotherapy with S-1 and Oxaliplatin
}

\author{
Masato Kondo $^{a}$ Shogo Nishino ${ }^{b}$ Daisuke Yamashita ${ }^{b}$ Satoshi Kaihara ${ }^{a}$ \\ aDepartment of Surgery, Kobe City Medical Center General Hospital, Kobe, Japan; \\ bDepartment of Pathology, Kobe City Medical Center General Hospital, Kobe, Japan
}

Keywords

Gastric cancer · Neoadjuvant chemotherapy · S-1 - Oxaliplatin · Pathological complete response

\begin{abstract}
The prognosis of locally advanced gastric cancer is poor even if radical gastrectomy with D2 lymphadenectomy is followed by adjuvant chemotherapy. Hence, neoadjuvant chemotherapy is performed to try to improve the prognosis, as it can significantly downstage the tumor and safely improve the R0 resection rate of patients. Herein, we report a case of locally advanced gastric cancer with pancreatic invasion and gastric outlet obstruction that showed a pathological complete response after neoadjuvant chemotherapy with S-1 and oxaliplatin (SOX). A 74-year-old man presented to our hospital with abdominal pain and pyloric stenosis. CT images revealed a cStage IVb, cT4b tumor in the pancreas, cN1, cM0. Therefore, we performed laparoscopic gastrojejunostomy, and the patient's oral intake improved after surgery; we then administered neoadjuvant chemotherapy with SOX on postoperative day 18 , without any surgical complications. After 3 courses of neoadjuvant chemotherapy, the patient underwent radical distal gastrectomy, thereby avoiding pancreatoduodenectomy. Histopathological examination of the resected sample revealed no residual cancer cells, indicating a pathological complete response. No recurrence has occurred for 1 year after surgery. Thus, neoadjuvant chemotherapy with SOX can help in tumor downstaging and may be a multipotent option for the treatment of locally advanced gastric cancer, such as cases with the invasion of other organs; this treatment can result in improved curability and avoid overinvasive surgery.
\end{abstract}




\section{Introduction}

Gastric cancer is among the most common causes of cancer-related death worldwide [1]. The prognosis of locally advanced gastric cancer is poor even if radical gastrectomy with D2 lymphadenectomy is followed by adjuvant chemotherapy [2]. Therefore, combined-modality therapy, such as neoadjuvant chemotherapy, is performed to improve the prognosis, as it can significantly downstage the tumor and safely improve the R0 resection rate of Asian patients [3]. Herein, we report a case of a locally advanced gastric cancer with pancreatic invasion and gastric outlet obstruction that showed a pathological complete response after neoadjuvant chemotherapy with S-1 and oxaliplatin (SOX).

\section{Case Presentation}

A 74-year-old man was admitted to our hospital, as he was unable to eat owing to pyloric stenosis caused by a large gastric cancer. Upper gastrointestinal endoscopy revealed a type 3 tumor in the region from the gastric angle to the pylorus (Fig. 1), and a biopsy indicated a poorly differentiated adenocarcinoma. CT images revealed that the gastric wall was remarkably thickened, and the tumor directly and widely invaded the duodenum and pancreatic head (Fig. 2). The carcinoembryonic antigen level was $3.7 \mathrm{ng} / \mathrm{mL}$, and the cancer antigen 19-9 level was $19.1 \mathrm{U} / \mathrm{mL}$. Laparoscopic gastrojejunostomy with incomplete transection of the stomach was performed to improve food passage, and neoadjuvant oxaliplatin $\left(130 \mathrm{mg} / \mathrm{m}^{2}\right)$ was administered on the 18th postoperative day in combination with $\mathrm{S}-1$ (80 $\mathrm{mg} / \mathrm{m}^{2} /$ day) for 14 days.

After 3 courses of preoperative chemotherapy as planned, the pyloric mass was not observed on FDG-PET (fluorodeoxyglucose-positron emission tomography), and FDG accumulation was negligible (Fig. 3).

Radical distal gastrectomy was performed without any complications. The lymph nodes with suspected pancreatic infiltration that were noted prior to neoadjuvant chemotherapy were scarred and could be dissected from the pancreatic head, thereby avoiding pancreatoduodenectomy, and gross R0 resection could be achieved. The operation time was $316 \mathrm{~min}$, and the amount of blood loss was $208 \mathrm{~mL}$. The patient was discharged from the hospital on the 7 th postoperative day, with good postoperative recovery.

Histopathological examination of the resected specimens from both the stomach and the dissected lymph nodes revealed no obvious cancer cells on hematoxylin and eosin staining, although fibrosis with lymphocytic infiltration was extensively observed (Fig. 4). Therefore, the patient showed a pathological complete response after neoadjuvant chemotherapy with SOX.

Fig. 1. Upper gastrointestinal endoscopy revealed a huge type 3 gastric tumor and pyloric stenosis.

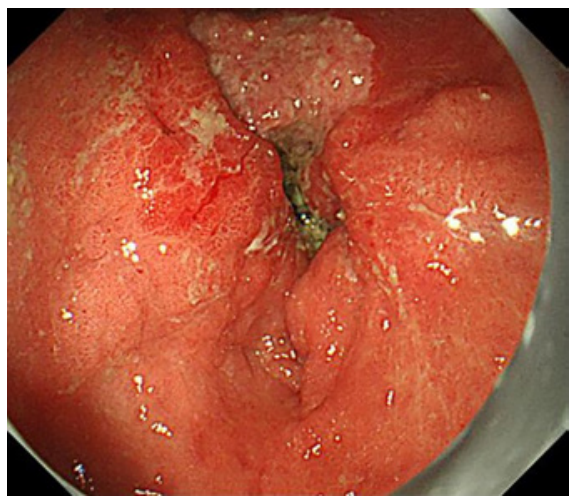



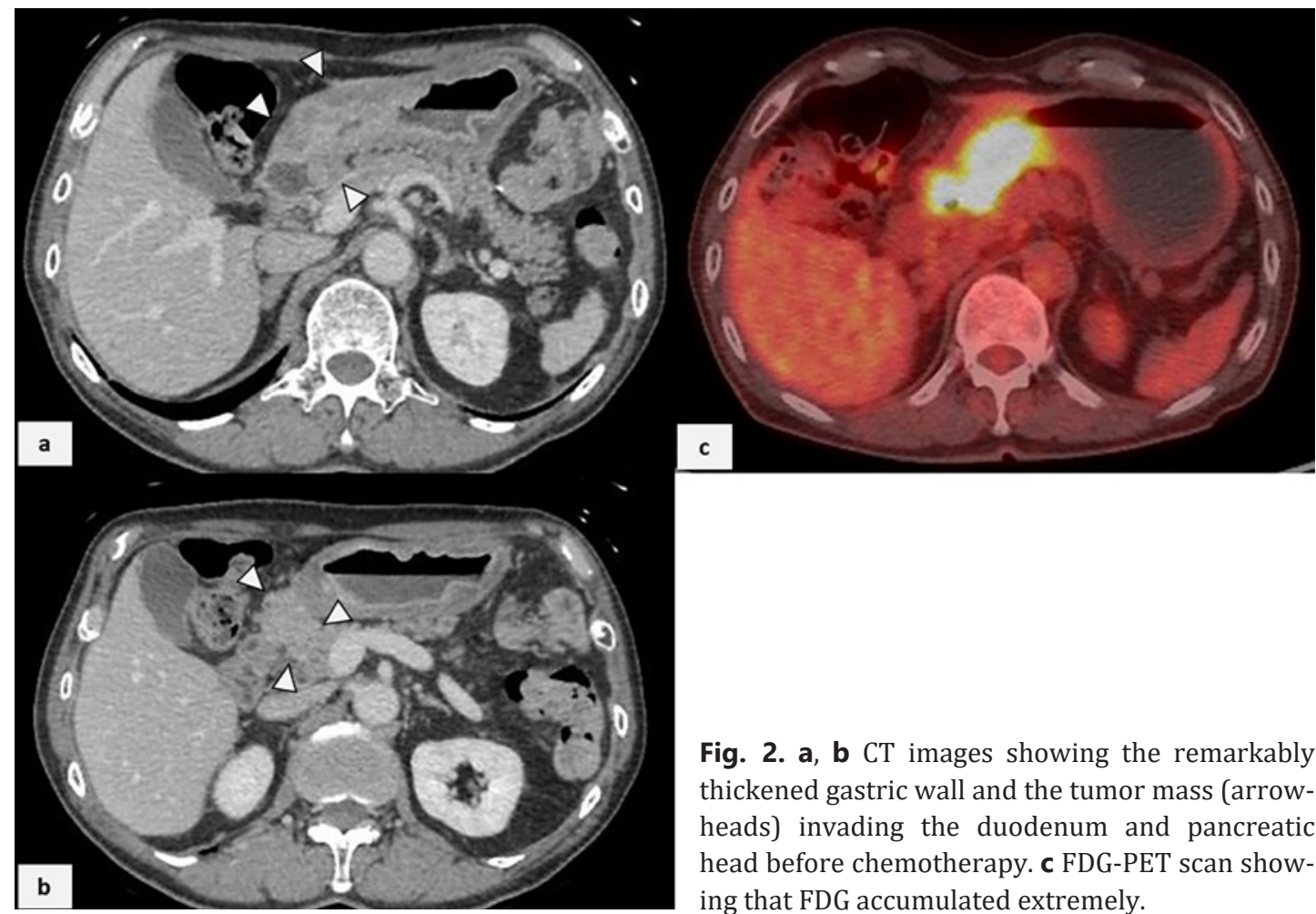

Fig. 2. a, b CT images showing the remarkably thickened gastric wall and the tumor mass (arrowheads) invading the duodenum and pancreatic head before chemotherapy. c FDG-PET scan showing that FDG accumulated extremely.

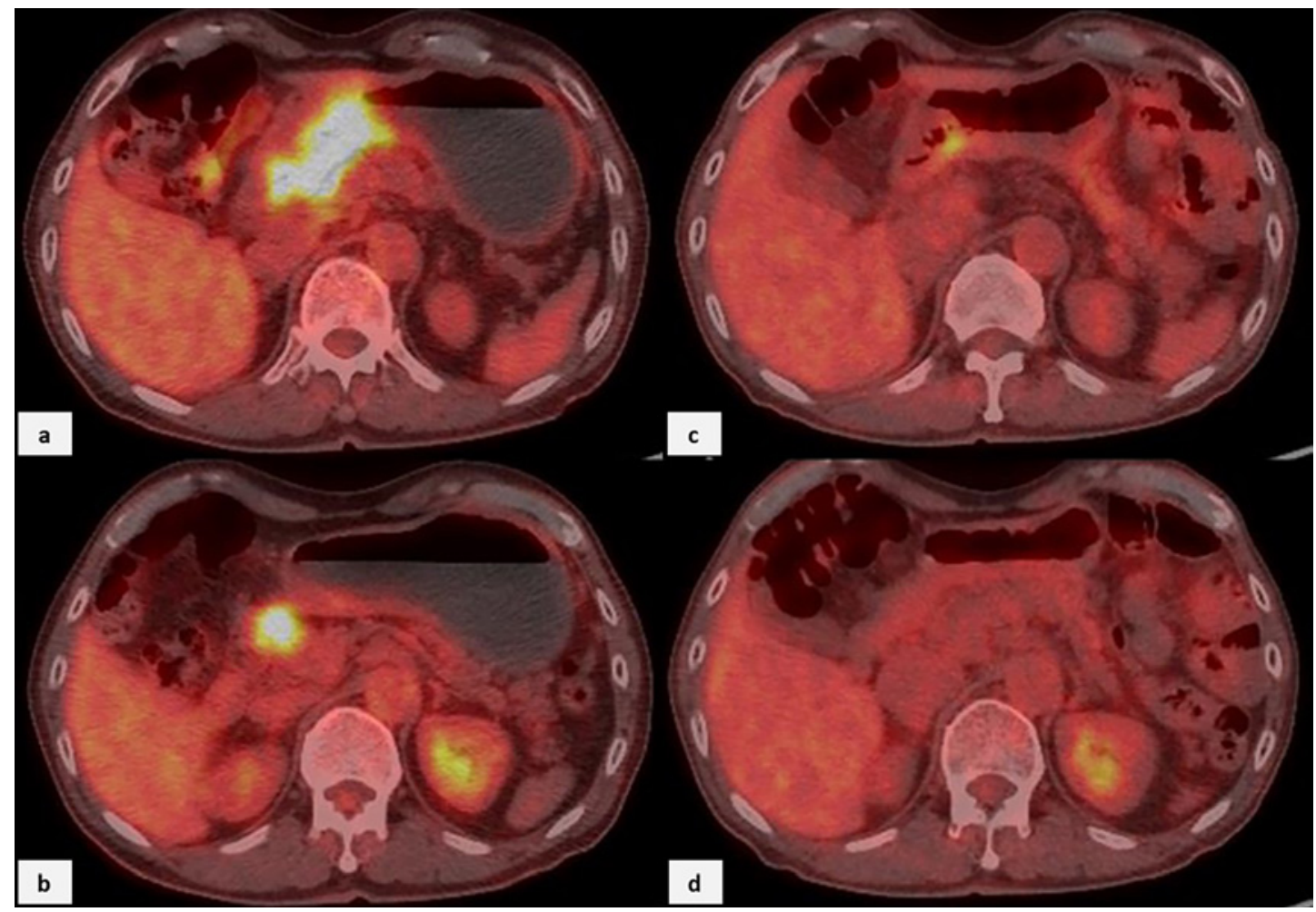

Fig. 3. FDG-PET scans showing that FDG accumulation had considerably decreased in the tumor mass after chemotherapy $(\mathbf{c}, \mathbf{d})$ compared to that observed before chemotherapy $(\mathbf{a}, \mathbf{b})$. 


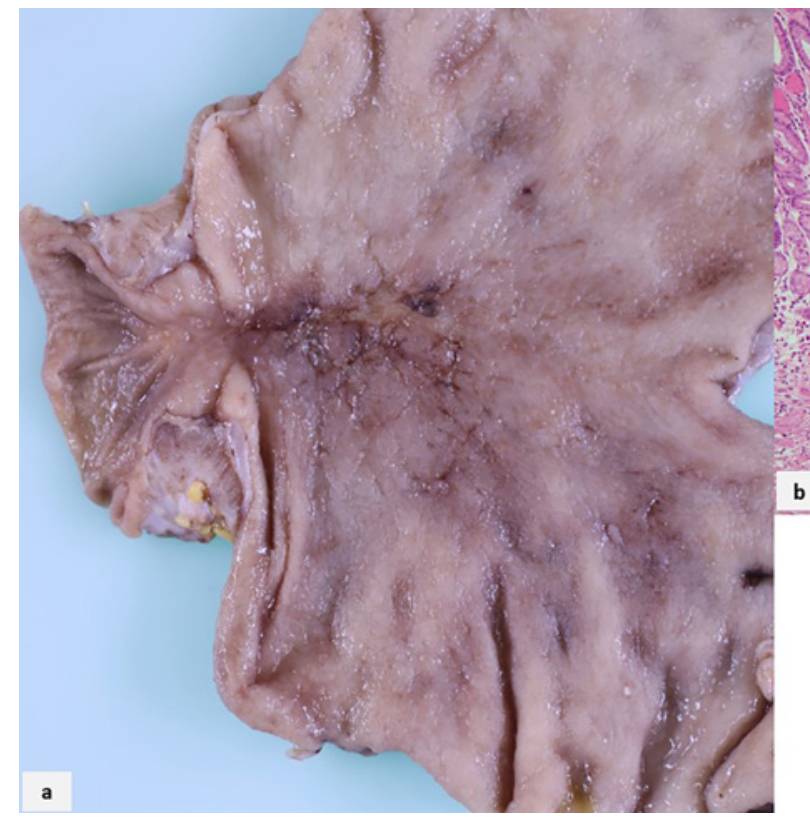

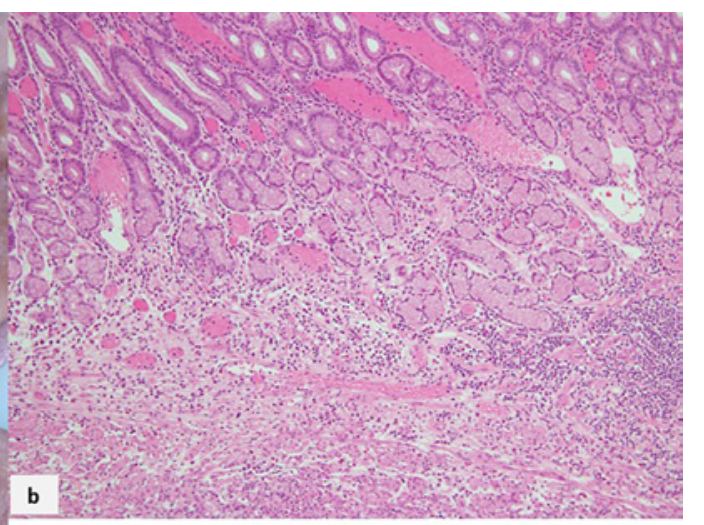

Fig. 4. a Resected specimen in which the pyloric lesion was scarred. b On histopathological examination after chemotherapy, no cancer cells were observed in the resected specimen, although fibrosis with lymphocytic infiltration was observed.

\section{Discussion}

Many patients with advanced gastric cancer experience recurrence after radical surgery followed by adjuvant chemotherapy, particularly patients with bulky nodal metastases or large type 3 or 4 tumors. Neoadjuvant chemotherapy seems to be one of the effective treatments for advanced gastric cancer. Although preoperative treatment is associated with increased downstaging, thereby increasing the R0 resection rates [4], currently, it is not clear whether neoadjuvant chemotherapy or postoperative adjuvant chemotherapy is better considering the prognosis $[5,6]$.

In the current case, there were no distant metastases or dissemination, and all the factors were indicators of performing resection. However, owing to the presence of duodenal and pancreatic invasion, pancreatoduodenectomy was performed as radical resection; it is a very invasive procedure and may have resulted in a decline in the postoperative quality of life, so it is possible that adequate adjuvant chemotherapy could not be performed. Hence, neoadjuvant chemotherapy was performed, as it would not only allow the patient the opportunity to undergo regular radical distal gastrectomy - avoiding overinvasive surgery - but also result in a complete response after chemotherapy with a sufficient dose, thereby resulting in a higher $\mathrm{R} 0$ resection rate. Thus, even if radical resection is possible, perioperative chemotherapy might improve the prognosis of patients with highly advanced gastric cancer, such as cT4b tumors with invasion to other organs.

The treatments for symptomatic pyloric stenosis caused by gastric cancer include stent insertion and an alimentary bypass. As we performed bypass surgery on the current patient, the nutritional status markedly improved, with an increase in oral intake, and adequate neoadjuvant chemotherapy could be administered easily. We first performed the modified Devine exclusion, as reported by Schantz et al. [7], with incomplete transection of the stomach as a gastric bypass to evaluate the tumor via endoscopic examination after chemotherapy. Then, after neoadjuvant chemotherapy, we only resected the distal side of the stomach and then performed gastrojejunostomy as part of the modified Devine exclusion in order to reduce surgical invasion as much as possible. 
Kondo et al.: CR of Gastric Cancer after Neoadjuvant Chemotherapy with SOX

\section{Conclusion}

Neoadjuvant chemotherapy with SOX resulted in tumor downstaging and might be a multipotent option for the treatment of locally advanced gastric cancer, such as tumors with the invasion of other organs, as it can improve the curative rate and avoid the performance of overinvasive surgery. Nevertheless, clinical trials that evaluate the effectiveness of perioperative chemotherapy in such cases of advanced gastric cancer are being conducted, and the results are awaited [8].

\section{Acknowledgement}

We would like to thank Editage (www.editage.com) for English language editing.

\section{Statement of Ethics}

Written informed consent for the publication of this paper was obtained from the patient.

\section{Disclosure Statement}

The authors declare that they have no conflict of interest to disclose.

\section{Funding Sources}

No funding was received for this case report.

\section{Author Contributions}

All authors contributed to the paper, including writing and revising it. All authors approved the final version of the manuscript.

\section{References}

1 Ferlay J, Soerjomataram I, Dikshit R, Eser S, Mathers C, Rebelo M, et al. Cancer incidence and mortality worldwide: sources, methods and major patterns in GLOBOCAN 2012. Int J Cancer. 2015 Mar 1;136(5):E359-86.

2 Noh SH, Park SR, Yang HK, Chung HC, Chung IJ, Kim SW, et al. Adjuvant capecitabine plus oxaliplatin for gastric cancer after D2 gastrectomy (CLASSIC): 5-year follow-up of an open-label, randomised phase 3 trial. Lancet Oncol. 2014 Nov;15(12):1389-96.

3 Xiong BH, Cheng Y, Ma L, Zhang CQ. An updated meta-analysis of randomized controlled trial assessing the effect of neoadjuvant chemotherapy in advanced gastric cancer. Cancer Invest. 2014 Jul;32(6):272-84.

4 Wang XZ, Zeng ZY, Ye X, Sun J, Zhang ZM, Kang WM. Interpretation of the development of neoadjuvant therapy for gastric cancer based on the vicissitudes of the NCCN guidelines. World J Gastrointest Oncol. 2020 Jan 15; 12(1):37-53.

5 Chen J, Ye Q, Huang F. Historical evolution and research progress of perioperative therapy of locally advanced gastric cancer [in Chinese]. Zhonghua Wei Chang Wai Ke Za Zhi. 2019 Feb;22(2):196-200.

6 Xue K, Ying X, Bu Z, Wu A, Li Z, Tang L, et al. Oxaliplatin plus S-1 or capecitabine as neoadjuvant or adjuvant chemotherapy for locally advanced gastric cancer with D2 lymphadenectomy: 5-year follow-up results of a phase II-III randomized trial. Chin J Cancer Res. 2018 Oct;30(5):516-25.

7 Schantz SP, Schickler W, Evans TK, Coffey RJ. Palliative gastroenterostomy for pancreatic cancer. Am J Surg. 1984 Jun 1;147(6):793-6.

8 Tokunaga M, Sato Y, Nakagawa M, Aburatani T, Matsuyama T, Nakajima Y, et al. Correction to: Perioperative chemotherapy for locally advanced gastric cancer in Japan: current and future perspectives. Surg Today. 2020 Jan;50(4):424-7. 